\title{
PRÁTICA ALIMENTAR DE ADOLESCENTES ${ }^{1}$
}

\section{ADOLESCENTS FEEDING PRACTICES}

\author{
Ana Maria Dianezi GAMBARDELLA² \\ Maria Fernanda Petroli FRUTUOSO ${ }^{3}$ \\ Claudia FRANCH ${ }^{3}$
}

RESUMO

Visando conhecer a prática alimentar de adolescentes, foi realizado inquérito alimentar com um grupo de 153 estudantes, de ambos os sexos, com idade média de 14,0 1 1,5 anos, utilizando questionário auto-aplicado. Foi efetuada análise qualitativa das três principais refeições diárias, comparando-se com padrão estabelecido. Verificou-se que o desjejum foi consumido por $45 \%$ (44\% meninos e $56 \%$ meninas), o almoço por $76 \%$ (36\% meninos e 64\% meninas) e o jantar por 53\% (40\% meninos e 60\% meninas). Observou-se que a constituição do jantar é basicamente, a mesma que a do almoço, notando-se porém a diminuição da ingestão de alimentos fonte de proteína e ferro, tais como a carne e o feijão, substituídos por alimentos fonte de proteína e cálcio (leite e derivados), o que remete a hipótese de que o lanche está tomando o lugar do jantar. O desjejum é a refeição mais negligenciada pelos adolescentes estudados. Faz-se necessário controlar a ingestão de alimentos entre as três refeições, pois esta poderá estar contribuindo de duas formas: favorecendo o consumo de nutrientes que poderiam estar deficientes ou, por outro lado, agravando quadros tais como obesidade entre outros.

Termos de indexação: hábitos alimentares, adolescência, estudantes, inqueritos nutricionais.

\section{ABSTRACT}

A study was carried out on a group of 153 students of both sexes, with an average age of $14.0 \pm 1.5$ years, using a self-applied questionnaire, aiming to discover the feeding practices of adolescents. A qualitative analysis of the three main daily meals (breakfast, lunch and dinner) in comparison with an established standard was made. It was found that $45 \%$ of the adolescents (44\% boys and 56\%, girls) took breakfast. Lunch was taken by $76 \%$ of the adolescents (36\% boys and $64 \%$ girls). With regard to dinner, taken by $53 \%(40 \%$ boys and $60 \%$ girls), it was found to be constituted, basically, of the same foodstuffs and/

(1) Trabalho apresentado no XVI Congresso Internacional de Nutrição, Montreal, Canadá, em 1997.

(2) Departamento de Nutrição da Faculdade de Saúde Pública da Universidade de São Paulo, Av. Dr. Arnaldo, 715, 01246-904, São Paulo, SP. E-mail: gambarde@usp.br.

(3) Pós-graduanda da Faculdade de Saúde Pública da Universidade de São Paulo. 
or preparations of their lunch, though with a reduction in food sources of protein and iron, such as meat and beans, replace by food sources of protein and calcium (milk and its products), which leads to the conclusion that snacks are taking the place of dinner. The breakfast is the most neglected meal by the adolescents studied. It is necessary to control the consumption of food between the three meals, because this may be contributing in two ways: by increasing the consumption of the nutrients that may otherwise be insufficient or, on the other hand, by aggravating problems such as obesity, among others.

Index terms: food habits, adolescence, students, nutrition surveys.

INTRODUÇÃO

A adolescência, do latim adolescere (crescer), é um período de várias mudanças que acontece entre os 10 e 20 anos de idade, marcado por transformações físicas aceleradas e características da puberdade, diferentes do crescimento e desenvolvimento que ocorrem em ritmo constante na infância. Essas alterações são influenciadas por fatores hereditários, ambientais, nutricionais e psicológicos (Organización..., 1965; Post \& Kemper, 1993).

Além do aspecto físico, há também mudanças sociais, quando o adolescente começa a adquirir independência e responsabilidades, e mudanças psicológicas, como o aumento da capacidade cognitiva e adaptações de personalidade, constituindo uma parte da população com características fisiológicas e psicológicas específicas (Lerner, 1994).

Todas as transformações da adolescência têm efeito sobre o comportamento alimentar, influenciado por fatores internos, auto-imagem, necessidades fisiológicas e saúde individual, valores, preferências e desenvolvimento psicossocial; e por fatores externos, hábitos familiares, amigos, valores e regras sociais e culturais, mídia, modismos, experiências e conhecimentos do indivíduo (Farthing, 1991).

A família é a primeira instituição que tem ação sobre os hábitos do indivíduo. É responsável pela compra e preparo dos alimentos em casa, transmitindo seus hábitos alimentares às crianças.

Os adolescentes tendem a viver o momento atual, não dando importância às consequiências de seus hábitos alimentares, que podem ser prejudiciais. Sabe-se que hábitos alimentares inadequados na infância e adolescência podem ser fatores de risco para doenças crônicas e obesidade (Anderson, 1991; Farthing, 1991; Bourne et al., 1994; Sargent et al., 1994 Anding et al., 1996).
Os adolescentes passam, gradativamente, maior tempo fora de casa, na escola e com os amigos que, também, influenciam na escolha dos alimentos e estabelecem o que é socialmente aceito.

É característica da alimentação desses jovens (e da vida moderna) o consumo de lanches e fast foods, entre as refeições (Bull, 1988; Farthing, 1991; Sargent et al., 1994; Andersen et al., 1995). Esta atitude pode ser justificada pela falta de tempo disponível para dedicar a uma refeição, preferências individuais, modismo e por ser uma refeição que pode ser feita com os amigos.

O impacto nutricional dos lanches e fast foods pode ser influenciado por alguns fatores como, a frequiência de consumo e valores nutricionais dos alimentos escolhidos. Tais preparações podem ser aceitáveis, quando parte de uma dieta adequada e balanceada mas, geralmente apresentam alta quantidade de energia e baixa quantidade de ferro, cálcio, vitamina A e fibras (Bull, 1988).

Muitas vezes, os adolescentes consomem refeições de modo irregular e tendem a "pular" refeições, principalmente o desjejum (Bull, 1988; Andersen et al., 1995). Isso é mais freqüente entre as meninas como forma de perder peso (Bull, 1988; Sargent et al., 1994; French et al., 1995). Em geral, apresentam dietas inadequadas em relação a vários nutrientes. Sargent (1994), observou baixo consumo de fibras, ferro e cálcio e alto consumo de proteínas e colesterol em estudantes da Carolina do Sul, Estados Unidos, bem como baixa ingestão de frutas e hortaliças cruas e cozidas. Encontra-se baixa ingestão de leite, frutas e hortaliças entre a população, de 15 a 64 anos, da África do Sul (Bourne et al., 1994). Segundo Jonhson et al. (1994), a raça e região são os fatores sócio-demográficos que mais afetam alimentação de adolescentes norte-americanos que, no geral, têm baixa ingestão de vitaminas e minerais. 
Estudos sobre a alimentação de grupos de adolescentes brasileiros, indica ocorrência de inadequação alimentar com carência de ingestão de produtos lácteos, frutas e hortaliças e excesso de açúcar e gordura (Gambardella, 1996). Lerner (1994), observou baixo consumo de alimentos fontes de vitamina $\mathrm{C}$ que poderiam aumentar a biodisponibilidade de ferro na dieta, uma vez que este mineral apresenta inadequado consumo entre a população.

Dada a importância indiscutível da alimentação é fundamental conhecer a prática (o que se faz) alimentar de adolescentes, objetivo deste estudo. Entende-se por prática, a atitude do indivíduo em relação a dado objeto e de uma atitude relacionada à fatores externos que envolvem esse objeto.

\section{MATERIAL E MÉTODOS}

\section{Casuística}

Foi realizado inquérito alimentar, utilizando questionário auto-aplicado (Gambardella, 1996), com um grupo de 153 adolescentes, voluntários (aproximadamente $25 \%$ do total de formulários distribuídos), de 11 a 18 anos de idade, de ambos os sexos, estudantes do primeiro grau, do período diurno, de seis escolas da rede estadual, pertencentes a mesma região de Santo André, SP. Nos questionários, que foram aplicados pelos professores das próprias escolas, perguntava-se "o que o indivíduo estava acostumado a comer", considerando nome da refeição, horário, local e os alimentos e/ou preparações consumidas. Solicitava-se, ainda, informar o "horário que está habituado dormir e acordar".

Como critério para a avaliação qualitativa da prática alimentar habitual desses estudantes, considerou adequada a dieta que apresentasse, no mínimo, três refeições diárias (desjejum, almoço e jantar), constituídas de alimentos fontes de nutrientes essenciais. Para a determinação das refeições padrão, levou-se em conta os alimentos e/ou preparações consumidas por adolescentes, tal como o observado por Priore (1996). Desse modo, a dieta padrão dos adolescentes ficou assim constituída:

- Desjejum padrão: deve conter alimentos fonte de cálcio e de energia. A fonte de cálcio seria representada por leite e derivados, principais fontes dietéticas desse mineral, bem como, de proteínas. A fonte energética, composta por pães e biscoitos, com algum tipo de acompanhamento, ou seja, tudo o que habitualmente se consome com pães e biscoitos: geléias, mel, margarinas, manteiga, maionese, queijos e frios.

- Almoço e jantar padrão: alimentos fonte de proteína, energia, vitaminas, minerais e de fibras. Foi considerada como fonte protéica, o feijão, carnes ou ovos, sendo as duas primeiras, também fontes dietéticas de ferro. A energética composta, basicamente, por cereais e tubérculos, tais como arroz, massas em geral e batata e, a de vitaminas, minerais e fibras por frutas e hortaliças.

A análise foi baseada no registro da presença ou ausência do alimento fonte, isto é, ponderou-se apenas a citação do alimento, pertencente à refeição padrão.

Procurou-se conhecer os horários de sono e vigília, bem como, aqueles referentes ao consumo de refeições.

\section{RESULTADOS E DISCUSSÃO}

\section{Desjejum padrão}

Verificou-se que $82 \%$ dos adolescentes realizavam o desjejum consumindo algum tipo de alimento.

O desjejum padrão foi relatado por $45 \%$ dos adolescentes, sendo 52\% do sexo masculino e o restante do feminino. A maioria desses (99\%) realizava esta refeição no próprio domicílio, entre 6he 11h30min.

Em relação ao sono e vigília, observou-se que os horários em que os estudantes acordam e dormem variam, respectivamente, das $5 \mathrm{~h}$ às $10 \mathrm{~h} 30 \mathrm{~min}$ e das $19 \mathrm{~h}$ às $0 \mathrm{~h} 30 \mathrm{~min}$, sendo que $63 \%$ dos adolescentes acordam entre $6 \mathrm{~h}$ e $6 \mathrm{~h} 30$ min e $65 \%$ dormem entre $22 \mathrm{~h}$ e $23 \mathrm{~h}$. Com base no horário de sono e vigília e nas informações sobre a realização de alguma refeição à noite (ceia), ou pela manhã (lanche), verificou-se que acordar tarde (após às 10h) não é justificativa para não realizar o desjejum.

Observou-se que $56 \%$ dos estudantes que não tomavam o desjejum, também não realizavam ceia e lanche matinal, permanecendo um longo período em jejum, ou seja, do jantar de um dia ao almoço do dia seguinte. Dos demais que não faziam o desjejum, 33\% consumiam um lanche pela manhã e $11 \%$ apontaram 
que ceiavam, o que pode contribuir para a diminuição desse período. A prática dos adolescentes não realizaremodesjejuminviabilizaa elevaçãodaglicemia necessária às atividades matinais, e uma possível deficiência de cálcio, que na população em estudo, é nessa refeição que se concentra o maior consumo de alimentos fontes desse mineral.

Estudo realizado com 1323 estudantes suíços, de 15 a 20 anos, mostrou que não tomavam o desjejum, $27 \%$ e $24 \%$ dos adolescentes de sexo feminino e masculino, respectivamente. Os motivos citados como justificativa para "pular" essa refeição foram a falta de tempo (34\%), não ter o hábito de fazê-lo (32\%) e não ter apetite (25\%). Para as meninas, não tomar o desjejum pode ser um resultado da motivação para a perda de peso, o que não se sabe é se, realmente, sentem-se inapetentes ou se é uma estratégica tentativa de emagrecimento. Verificou, ainda, que dois terços levavam menos de quinze minutos para realizá-lo (Cavadini, 1996).

Práticas alimentares adequadas desde a infância podem diminuir um possível fator de risco para algumas doenças, como por exemplo, a osteoporose. A ingestão adequada de cálcio durante a vida toda auxilia no combate à osteoporose, distúrbio metabólico onde ocorre diminuição de massa óssea (Simopoulos \& Galli, 1993).

Na Tabela 1, 34\% dos indivíduos estudados nãoconsomemleitee derivadosnodesjejum. Oconsumo de leite e derivados garante a ingestão de cálcio, mineral essencial na constituição óssea do indivíduo. Embora não seja objetivo realizar uma análise quantitativa da ingestão de cálcio, é importante ressaltar que $63 \%$ das meninas ingerem alimentos fonte desse mineral no desjejum. Tal consumo é fundamental para as reservas orgânicas de cálcio e possível prevenção da osteoporose.

Denis et al. (1990), estudando a dieta de 283 adolescentes franceses constatou que $44 \%$ consumiam leite com chocolate e $24 \%$, leite com café. Diferentemente dos estudantes de Santo André, que têm como prática o consumo de leite (24\%) e leite com café $(34 \%)$. Somente $5 \%$ dos adolescentes consomem leite com chocolate.

Observa-se na Tabela 2 que $45 \%$ e $40 \%$ de meninos e meninas, respectivamente, consomem diariamente pães e/ou biscoitos com margarina, enquanto que $23 \%$ dos meninos e $31 \%$ das meninas não ingerem esses alimentos no desjejum.

Tabela 1. Consumo de alimentos e/ou preparações fontes de cálcio no desjejum, segundo sexo. Santo André, São Paulo (1992).

\begin{tabular}{|c|c|c|c|c|c|c|}
\hline \multirow{2}{*}{ Alimentos e/ou preparações } & \multicolumn{2}{|c|}{ Meninos } & \multicolumn{2}{|c|}{ Meninas } & \multicolumn{2}{|c|}{ Total } \\
\hline & $\mathrm{n}$ & $\%$ & $\mathrm{n}$ & $\%$ & $\mathrm{n}$ & $\%$ \\
\hline Leite, leite com café ou chocolate ou fruta & 39 & 65 & 56 & 62 & 95 & 63 \\
\hline Queijo & 3 & 5 & 1 & 1 & 4 & 3 \\
\hline Não consomem & 18 & 30 & 34 & 37 & 52 & 34 \\
\hline Total & 60 & 100 & 91 & 100 & 151 & 100 \\
\hline
\end{tabular}

Tabela 2. Consumo de alimentos energéticos e acompanhamentos no desjejum, segundo sexo. Santo André, São Paulo (1992).

\begin{tabular}{|c|c|c|c|c|c|c|}
\hline \multirow{2}{*}{ Alimentos e/ou preparações } & \multicolumn{2}{|c|}{ Meninos } & \multicolumn{2}{|c|}{ Meninas } & \multicolumn{2}{|c|}{ Total } \\
\hline & $\mathrm{n}$ & $\%$ & $\mathrm{n}$ & $\%$ & $\mathrm{n}$ & $\%$ \\
\hline Pão e/ou biscoito & 14 & 23 & 23 & 25 & 37 & 24 \\
\hline Pão e frios & 5 & 9 & 4 & 4 & 9 & 6 \\
\hline Pão e/ou biscoito e margarina & 27 & 45 & 36 & 40 & 63 & 42 \\
\hline Não consomem & 14 & 23 & 28 & 31 & 42 & 28 \\
\hline Total & 60 & 100 & 91 & 100 & 151 & 100 \\
\hline
\end{tabular}


A ingestão de infusões, chá e café, foi referida por $15 \%$ dos adolescentes, sendo $6 \%$ dos meninos e $9 \%$ das meninas.

Observou-se que o consumo de frutas in $n a-$ tura e suco de frutas nessa refeição é de $7 \%$ dos adolescentes ( $2 \%$ dos meninos e $5 \%$ das meninas).

\section{Almoço e jantar padrão}

Verificou-se o almoço padrão em $76 \%$ e, o jantar padrão em 53\% dos adolescentes. Informaram consumí-las em casa, $99 \%$ no almoço, tomado entre 11 e $14 \mathrm{~h}$ e, $98 \%$ no jantar, consumido entre $17 \mathrm{~h}$ e $22 \mathrm{~h} 30 \mathrm{~min}$
Na Tabela 3, observa-se que $87 \%$ e $80 \%$ dos meninos e meninas, respectivamente, consomem carnes (fonte de proteína animal) no almoço. Com relação ao feijão (proteína vegetal) constatou-se que $85 \%$ e $75 \%$ dos estudantes o consomem, no almoço e jantar, respectivamente. Deve ser lembrado que as proteínas são fundamentais na construção e manutenção dos tecidos corpóreos, participando também do transporte de substâncias e homeostase corpórea (Mahan \& Arlin, 1995). O ferro é parte de enzimas relacionadas com a respiração celular e imprescindível no transporte de oxigênio e gás carbônico. Sua deficiência leva à anemia, que tem como um dos principais sintomas a fadiga.

Tabela 3. Consumo de alimentos fontes de proteína no almoço e jantar, segundo sexo. Santo André, São Paulo (1992).

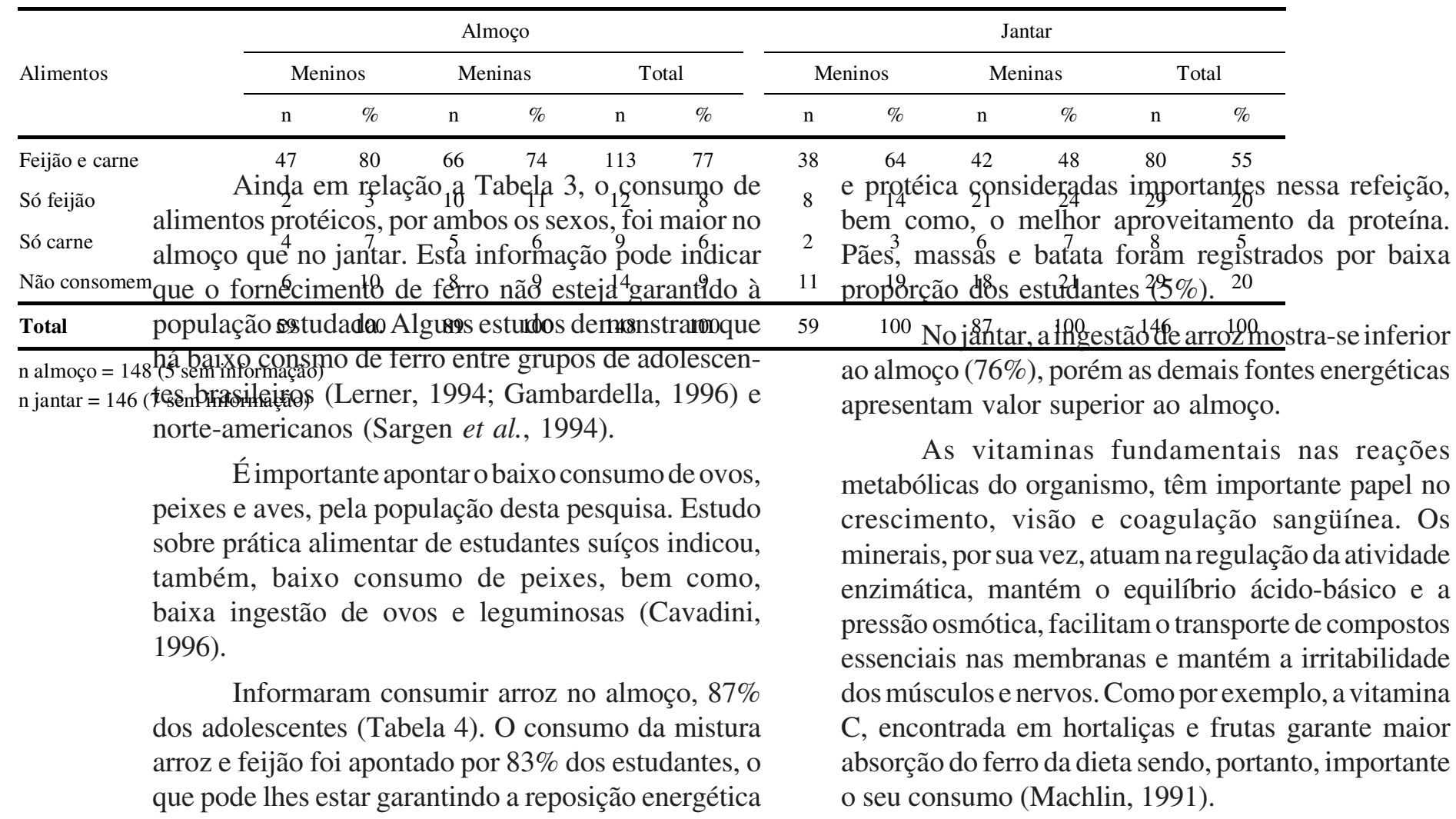


Tabela 4. Consumo de alimentos energéticos no almoço e jantar, segundo sexo. Santo André, São Paulo (1992).

\begin{tabular}{|c|c|c|c|c|c|c|c|c|c|c|c|c|}
\hline \multirow{3}{*}{ Alimentos } & \multicolumn{6}{|c|}{ Almoço } & \multicolumn{6}{|c|}{ Jantar } \\
\hline & \multicolumn{2}{|c|}{ Meninos } & \multicolumn{2}{|c|}{ Meninas } & \multicolumn{2}{|c|}{ Total } & \multicolumn{2}{|c|}{ Meninos } & \multicolumn{2}{|c|}{ Meninas } & \multicolumn{2}{|c|}{ Total } \\
\hline & $\mathrm{n}$ & $\%$ & $\mathrm{n}$ & $\%$ & $\mathrm{n}$ & $\%$ & $\mathrm{n}$ & $\%$ & $\mathrm{n}$ & $\%$ & $\mathrm{n}$ & $\%$ \\
\hline Pão & 2 & 3 & 2 & 2 & 4 & 3 & 5 & 8 & 4 & 5 & 9 & 6 \\
\hline Massas & 2 & 3 & 0 & 0 & 2 & 1 & 2 & 3 & 6 & 7 & 8 & 5 \\
\hline Batata & 0 & 0 & 1 & 1 & 1 & 1 & 0 & 0 & 1 & 1 & 1 & 1 \\
\hline Arroz & 51 & 87 & 78 & 88 & 129 & 87 & 44 & 75 & 67 & 77 & 111 & 76 \\
\hline Não consomem & 4 & 7 & 8 & 9 & 12 & 8 & 8 & 14 & 9 & 10 & 17 & 12 \\
\hline Total & 59 & 100 & 89 & 100 & 148 & 100 & 59 & 100 & 87 & 100 & 146 & 100 \\
\hline
\end{tabular}

$\mathrm{n}$ almoço $=148$ (5 sem informação)

$\mathrm{n}$ jantar $=146$ (7 sem informação)

Na Tabela 5, verifica-se que o consumo de hortaliças e frutas no almoço, fonte de vitaminas, minerais e fibras, foi registrado por $78 \%$ dos adolescentes. No entanto, no jantar, o consumo destes alimentos foi referido por $58 \%$.

Nota-se, surpreendentemente, que há maior consumo de hortaliças em relação às frutas. O esperado seria encontrar maior ingestão de frutas devido a facilidade de consumo em relação as hortaliças. Algumas frutas são consumidas imediatamente, enquanto as hortaliças precisam ser lavadas e preparadas para o consumo. Tal achado não vai de encontro a outros estudos como Andersen (1995), que estudando o padrão da alimentação de adolescentes da Noruega, constatou que a maior ingestão era de frutas e não de hortaliças.

Tabela 5. Consumo de hortaliças e frutas no almoço e jantar, segundo sexo. Santo André, São Paulo (1992).

\begin{tabular}{|c|c|c|c|c|c|c|c|c|c|c|c|c|}
\hline \multirow{3}{*}{ Alimentos } & \multicolumn{6}{|c|}{ Almoço } & \multicolumn{6}{|c|}{ Jantar } \\
\hline & \multicolumn{2}{|c|}{ Meninos } & \multicolumn{2}{|c|}{ Meninas } & \multicolumn{2}{|c|}{ Total } & \multicolumn{2}{|c|}{ Meninos } & \multicolumn{2}{|c|}{ Meninas } & \multicolumn{2}{|c|}{ Total } \\
\hline & $\mathrm{n}$ & $\%$ & $\mathrm{n}$ & $\%$ & $\mathrm{n}$ & $\%$ & $\mathrm{n}$ & $\%$ & $\mathrm{n}$ & $\%$ & $\mathrm{n}$ & $\%$ \\
\hline hortaliças & 41 & 70 & 64 & 72 & 105 & 71 & 31 & 53 & 45 & 52 & 76 & 52 \\
\hline suco de frutas & 3 & 5 & 5 & 6 & 8 & 6 & 3 & 5 & 3 & 3 & 6 & 4 \\
\hline fruta in natura & 0 & 0 & 2 & 2 & 2 & 1 & 0 & 0 & 3 & 3 & 3 & 2 \\
\hline não consomem & 15 & 25 & 18 & 20 & 33 & 22 & 25 & 42 & 36 & 42 & 61 & 42 \\
\hline Total & 59 & 100 & 89 & 100 & 148 & 100 & 59 & 100 & 87 & 100 & 146 & 100 \\
\hline
\end{tabular}

$\mathrm{n}$ almoço $=148$ ( 5 sem informação)

$\mathrm{n}$ jantar $=146$ (7 sem informação)

Observa-se que o consumo de frutas e hortaliças (fontes vitamínicas) entre os adolescentes do sexo feminino que no masculino. É importante ressaltar que os meninos representam um grupo mais suscetível às doenças cardiovasculares que podem ser prevenidas com o adequado consumo de hortaliças e frutas (Nobre \& Furtado, 1994).
Há maior consumo de pão e acompanhamentos no jantar, quando comparado ao almoço (Tabela 6). Isto deve-se a troca entre uma refeição completa por outra, mais rápida, composta por pão, acompanhamento e bebida. Percebe-se também, menor consumo de fontes protéicas no jantar. No almoço, 
$2 \%$ dos adolescentes consomem sopa ou "misturas" (preparações que acompanham o arroz e feijão). No jantar, 6\% ingerem essas preparações e 3\% consomem sanduíches. Ainda que as proporções sejam baixas, esse consumo é importante, uma vez que essas três preparações podem conter alimentos como carnes, hortaliças e cereais. A ingestão de refrigerantes apontada, no almoço por $12 \%$ e no jantar por $8 \%$ dos adolescentes, indica aumento da ingestão energética, podendo levar à obesidade.

Tabela 6. Consumo de outros alimentos e/ou preparações no almoço e jantar. Santo André, São Paulo, 1992.

\begin{tabular}{|c|c|c|c|c|c|c|}
\hline \multirow{2}{*}{ Alimentos e/ou preparações } & \multicolumn{2}{|c|}{ Almoço } & \multicolumn{2}{|c|}{ Jantar } & \multicolumn{2}{|c|}{ Total } \\
\hline & $\mathrm{n}$ & $\%$ & $\mathrm{n}$ & $\%$ & $\mathrm{n}$ & $\%$ \\
\hline Pão com manteiga & 5 & 4 & 8 & 5 & 13 & 4 \\
\hline Leite e derivados & 1 & 1 & 7 & 5 & 8 & 3 \\
\hline Sopa & 2 & 1 & 6 & 4 & 8 & 3 \\
\hline Sanduíche & 0 & 0 & 4 & 3 & 4 & 1 \\
\hline "Mistura" & 2 & 1 & 3 & 2 & 5 & 2 \\
\hline Refrigerante & 18 & 12 & 12 & 8 & 30 & 10 \\
\hline Não consomem & 120 & 81 & 106 & 73 & 226 & 77 \\
\hline Total & 148 & 100 & 146 & 100 & 294 & 100 \\
\hline
\end{tabular}

$\mathrm{n}$ almoço $=148$ (5 sem informação)

$\mathrm{n}$ jantar $=146$ (7 sem informação)

A ingestão de pelo menos duas refeições-padrão foi observada em $33,0 \%$ dos estudantes, $38,0 \%$ meninos e $62,0 \%$ meninas (Tabela 7). Três refeições padrão foram informadas por $17,0 \%$ dos adolescentes, dos quais $50 \%$ de meninos, proporção baixa, também encontrada em outros estudos. Inquérito alimentar realizado com estudantes espanhóis, por meio de registro de três dias constatou que apenas $1,6 \%$ consumiam dieta saudável, em um ou dois dias de estudo, e o restante em nenhum dos três dias analisados (Boneu et al., 1994).

Tabela 7. Número de refeições padrão consumidas, segundo sexo. Santo André, São Paulo, 1992.

\begin{tabular}{|c|c|c|c|c|c|c|}
\hline \multirow{2}{*}{ Número de refeições padrão } & \multicolumn{2}{|c|}{ Meninos } & \multicolumn{2}{|c|}{ Meninas } & \multicolumn{2}{|c|}{ Total } \\
\hline & $\mathrm{n}$ & $\%$ & $\mathrm{n}$ & $\%$ & $\mathrm{n}$ & $\%$ \\
\hline Uma & 20 & 33 & 28 & 30 & 48 & 31 \\
\hline Duas & 19 & 31 & 31 & 34 & 50 & 33 \\
\hline Três & 12 & 20 & 14 & 15 & 26 & 17 \\
\hline Não consomem & 10 & 16 & 19 & 21 & 29 & 19 \\
\hline Total & 61 & 100 & 92 & 100 & 153 & 100 \\
\hline
\end{tabular}

\section{CONCLUSÃO}

Constatou-se que $45 \%$ dos adolescentes apresentaram desjejum segundo o padrão estabelecido, sendo $44 \%$ meninos e $56 \%$ meninas, garantindo a ingestão de fontes de cálcio e de energia, consideradas importantes nessa refeição. 
O almoço, estabelecido como padrão foi encontrado em $76 \%$ dos adolescentes, sendo $36 \%$ meninos e $64 \%$ meninas. Ojantar padrão foi consumido por $53 \%$ dos estudantes, sendo $40 \%$ e $60 \%$ do sexo masculino e feminino, respectivamente.

A prática alimentar dos adolescentes estudados é inadequada já que há baixo consumo de hortaliças e frutas, principalmente no jantar, além de inadequado consumo de cálcio. Há, também, menor consumo de alimentos fonte de energia e proteína no jantar, em relação ao almoço. O almoço constituiu-se na refeição que a maior parte dos adolescentes ingeriram em conformidade com o padrão estabelecido, ao contrário do desjejum que foi a mais negligenciada, seguida pelo jantar. É preciso conhecer o consumo alimentar entre as três principais refeições e sua importância na dieta de adolescentes.

\section{REFERÊNCIASBIBLIOGRÁFICAS}

ANDERSEN, L.F., NES, M., SANDSTAD, B., BJORNEBOE, G-E., DREVON, C.A. Dietary intake among Norwegian adolescents. European Journal of Clinical Nutrition, London, v.49, n.8, p.555-564, 1995.

ANDERSON, J.J.B. The status of adolescent nutrition. Nutrition Today, Baltimore, v.26, n.2, p.7-10, 1991.

ANDING, J.D., KUBENA, K.S., McINTOSH, W.A., O'BRIEN, B. Blood lipids, cardiovascular fitness, obesity, and blood pressure: the presence of potential coronary heart disease risk factors in adolescents. Journal of the American Dietetic Association, Chicago, v.96,n.3, p.238-242, 1996.

BONEU, M., GÓMEZ-QUIRANTE, A., FEIJÓO, N., MARTOS, C., FOZ, G. Hábitos alimentarios y frecuencia de consumo de alimentos en los escolares de octavo de EGB de un Área Básica de Salud de Mataró. Atención Primaria, Barcelona, v.14, n.2, p.591-595, 1994.

BOURNE, L.T., LANGENHOVEN, M.L., STEYN, K., JOOSTE, P.L., NESAMVUNI, A.E., LAUBSCHER, J.A. The food and meal pattern in the urban African population of the Cape Peninsula, South Africa: the BRISK Study. Central African Journal of Medicine, Harare, v.40, n.6, p.140-148, 1994.
BULL, N.L. Study of the dietary habits, foods consumption and nutrients intakes of adolescents and young adults. World Review of Nutrition and Dietetics, Basel, v.57,p.24-74, 1988.

CAVADINI, C. Dietary habits in adolescence: contribution of snacking. In: FEEDING from toddlers to adolescence. Philadelphia : Lippincott-Raven Publishers, 1996. (Nestlé Nutrition Workshop Series, 37).

DENIS, C., MICHAUD, C., DENIS, G., MEJEAN, L. Le petit-dejeuner: habitudes alimentaires d'adolescents scolarisés. Cahiers de Nutrition et de Diététique, Paris, v.25, n.6, p.432-435, 1990.

FARTHING, M.C. Current eating patterns of adolescents in the United States. Nutrition Today, Baltimore, v.26, n.2,p.35-39, 1991.

FRENCH, S.A., STORY, M., DOWNES, B., RESNICK, M.D., BLUM, R.W. Frequent dieting among adolescents: psychosocial and health behavior correlates. American Journal of Public Health, Washington DC, v.85, n.5, p.695-701, 1995.

GAMBARDELLA, A.M.D. Adolescentes, estudantes de período noturno: como se alimentam e gastam suas energias. São Paulo, 1996. p.42-60. Tese (Doutorado) - Faculdade de Saúde Pública da USP, 1996.

JONHSON, R.K., JONHSON, D.G., WANG, M.Q., SMICIKLAS-WRIGHT, H., GUTHRIE, H.A. Characterizing nutrient intakes of adolescents by sociodemographic factors. Journal of Adolescent Health, New York, v.15, n.2, p.149-154, 1994.

LERNER, B.R. Alimentação e a anemia carencial em adolescentes. São Paulo, 1994. p.26-77. Tese (Doutorado) - Faculdade de Saúde Pública da USP, 1994.

MACHLIN, L.J. Handbook of vitamins. 2.ed. New York : MarcelDekker, 1991.p.195-232.

MAHAN, L.K., ARLIN, M.T. Krause: alimentos, nutrição e dietoterapia. 8.ed. São Paulo : Roca, 1995. p.57-70.

NOBRE, F., FURTADO, M.R. II Conselho brasileiro para o tratamento da hipertensão arterial. Revista da Associação Médica Brasileira, São Paulo, v.40, n.4, p.247-261, 1994.

ORGANIZACIÓN MUNDIAL DE LA SALUD. Problemas de salud de la adolescencia. Genebra, 1965. 29p. (OMS - Serie de Informes Técnicos, 308). 
POST, G.B., KEMPER H.C.G. Nutrient intake and biological maturation during adolescence. The Amsterdam growth and health longitudinal study. European Journal of Clinical Nutrition, London, v.47,n.6, p.400-408, 1993.

PRIORE, S. E. Perfil nutricional de adolescentes do sexo masculino residentes em favelas. São Carlos : UFSCar, 1996.p.51-65.

SARGENT, R.G., KEMPER, K.A., SCHULKEN, E. Dietary behaviors of South Carolina adolescents.
Journal of the South Carolina Medical Association, Columbia, v.90, n.6, p.263-269, 1994.

SIMOPOULOS, A.P., GALLI, C. Osteoporosis: nutritional aspects. World Review of Nutrition and Dietetics, Basel,v.73, 1993.

Recebido para publicação em 3 de setembro de 1997 e aceito em 29 de junho de 1998. 